\title{
In-situ Semantic 3D Modeling
}

Aditya Sankar

University of Washington

Seattle, WA, USA

aditya@cs.washington.edu
Permission to make digital or hard copies of part or all of this work for personal or classroom use is granted without fee provided that copies are not made or distributed for profit or commercial advantage and that coples bear this notice and the full citation For the list page. Copylight tor Chrd-party components of his work must be honored. For all other uses, contact the Owner/Auhor. Copyright is held by the Mobll

\begin{abstract}
Semantic 3D models of indoor scenes enable compelling interior design applications such as remodeling, refurnishing and rearrangement of furniture. However, creating these models is still a challenging task. Most existing approaches are designed to work ex-situ or out of context, and rely on the modeler's memory, photographs or measurements from the scene.

We propose a novel in-situ, mobile capture system that leverages quick and easy semantic input from the user and offloads tedious reconstruction and modeling tasks to the computer. In this way, our system combines the advantages of automatic and manual CAD based methods to significantly reduce modeling time and effort. Our approach runs on commodity mobile devices and can potentially scale to a much larger audience of casual mobile phone users.
\end{abstract}

\section{Author Keywords}

Interactive 3D Modeling; Sketching; Mobile Augmented Reality; Algorithms; Design; Human Factors

\section{ACM Classification Keywords}

H.5.2 [[Information Interfaces and Presentation]]: User Interfaces - Graphical user interfaces (GUI); I.3.6 [Computer Graphics]: Methodology and Techniques - Interaction techniques 


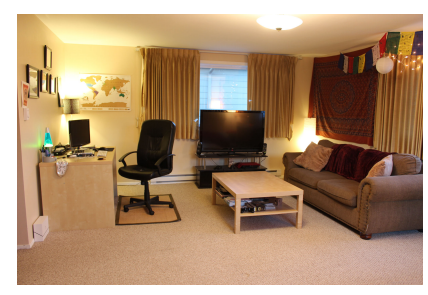

Figure 1: A photograph of an indoor scene. The goal is to capture it's digital likeness in an efficient manner.

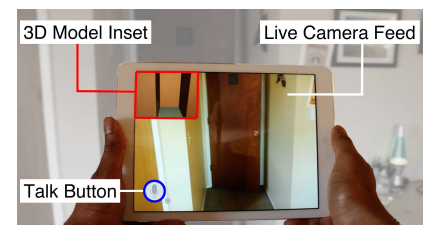

Figure 2: Our mixed-reality modeling interface.

\section{Research Goals, Questions and Approach}

This proposal introduces a mobile solution to easily capture representative semantic models of indoor scenes. My research seeks to find techniques that engage the user in an in-situ, interactive manner, to overcome sensory and computational limitations of the mobile platform.

A key idea is that we combine basic user input (annotations, sketches and voice commands) and 3D reconstruction algorithms, in a way that leverages the strengths of the human and the mobile computer. The system is designed to work in-situ, runs in real-time on a commodity smartphone or tablet without any additional hardware and allows users to create useful semantic reconstructions of their homes and offices, in a matter of minutes. The resulting 3D model captures walls, furniture, doors, windows, artwork etc. and is usable for interior design applications.

Our research indicates that the in-situ approach is on average faster than using traditional ex-situ desktop CAD modeling software, with some tradeoffs made on accuracy.

\section{Research Conducted So Far}

First, to capture the initial floor plan layout of the scene, we devised a smartphone application [1] to capture panoramic imagery from various rooms in an indoor scene. We then enlist the user to annotate wall edges and doors in each panorama. We then employ a Manhattan-world assumption and use constraint minimization to determine a floor-plan layout of the scene. The result is an interactive visual tour of the scene, augmented with a overhead map.

Once we have an 'empty' floor-plan layout, we extrude it into 3D and use a sketch based modeling interface (2) and voice commands [2] that allow the user to capture the contents of the real scene. In this way, the user can populate the 3D model with furniture, artwork, doors, windows etc., that closely resemble the appearance and position of objects in the real scene. The resulting semantic 3D mode enables the user to rearrange/replace/remove furniture swiftly, within the mobile application itself.

\section{Remaining Research and Expected Results}

The goal for the final phase of my doctoral work is to extend our system to capture more complete 3D models of complex scenes that match the layout and appearance of the scene with greater accuracy. In particular:

Larger Object Database: Freely available online 3D model repositories contain thousands of models. Indexing these models, coupled with efficient search would enable the accurate capture of a wider variety of scenes.

More Automation: User input can be error prone. Using computer vision techniques for recognition of features can mitigate this source of error.

Full-Floor Reconstruction: We would like to enable seamless capture of an entire floor and render it in a stylized manner, by shortening occluding walls.

New Class of Mobile Devices: Finally, we would like to explore how in-situ modeling interactions will evolve when used with emerging head-mounted devices.

\section{REFERENCES}

1. Aditya Sankar and Steven Seitz. 2012. Capturing Indoor Scenes with Smartphones. In Proceedings of the 25th Annual ACM Symposium on User Interface Software and Technology (UIST '12). ACM, 403-412. DOI: http://dx.doi.org/10.1145/2380116.2380168

2. Aditya Sankar and Steven Seitz. 2016. In Situ CAD Capture. In Proceedings of 18th International Conference on Human-Computer Interaction with Mobile Devices and Services (MobileHCl '16). ACM. DOI : http://dx.doi.org/10.1145/2935334.2935337 